\title{
Determination of Oxidative Stress Levels and Some Antioxidant Activities in Acute and Chronic Renal Failure Patients
}

\author{
Seerwan Hamadameen SULAIMAN ${ }^{1}$, Halit DEMIIR ${ }^{1}$, Yasemin Usul Soyoral ${ }^{2}$, Canan DEMİR ${ }^{3}$ \\ ${ }^{I}$ Van YuzuncuYll University, Faculty of Science Department of Chemistry, Van-Turkey \\ ${ }^{2}$ Van YuzuncuYll University, Faculty of Medicine Department of Nephrology, Van-Turkey \\ ${ }^{3}$ Van YuzuncuYll University, Vocational School of Health Services, Van-Turkey
}

*Corresponding Author: Seerwan Hamadameen SULAIMAN, Van YuzuncuYll University, Faculty of Science Department of Chemistry, Van-Turkey

\begin{abstract}
:
Aim: The aim of this study is to determine the relationship between superoxide dismutase (SOD), reduced glutathione (GSH), and catalase (CAT), and malondialdehyde (MDA) level in acute kidney failure and chronic kidney diseases.
\end{abstract}

Material and Methods: Biochemical parameters were determined from serum samples taken from acute and
chronic renal failure patients. Superoxide dismutase (SOD), reduced glutathione (GSH), catalase (CAT) and
malondialdehydeacid (MDA) levels were determined spectrophotometrically.

Results: The SOD activity of patient groups was significantly founded lower than that of healthy control group in acute and chronic renal failure $(p<0.05)$. In addition, serum GSH and CAT activities were significantly lower in the patient group compared to the control group $(p<0.05)$.

Conclusion: Superoxide dismutase (SOD), reduced glutathione (GSH), catalase (CAT) and malondialdehyde acid (MDA) levels may play an important role in the etiopathogenesis of acute and chronic renal failure patients. This study shows that the oxidative stress have strong influence on the cellular damage, and tissue in acute and chronic renal failure patients.This study is the first one to show the relationships of MDA, SOD, CAT and GSH in acute kidney failure and chronic kidney diseases.

Keywords: Acute renal failure, Chronic renal failure, CAT, GSH, MDA, SOD.

\section{INTRODUCTION}

Some conditions that may impact the function of the kidney include infections, kidney stones, acute kidney injury (AKI) and chronic kidney disease (CKD). When the kidneys are damaged or diseased, they can lose their ability to perform these vital functions suddenly or slowly, resulting in waste and fluid build-up and abnormal hormonal control of blood pressure and mineral homeostasis (1). Kidney diseases are mainly classified into two types, either acute kidney injury (AKI) or chronic kidney disease (CKD). AKI is characterized by sudden and sometimes fatal loss of function in the kidneys resulting in the accumulation of nitrogen metabolism (urea) and creatinine end products and reduced urine production or both (2).Kidney diseases are generally "silent diseases," without any apparent early symptoms most often. Most patients with kidney disease are unaware of the high risk of kidney failure that may require dialysis or transplantation, as well as cardiovascular diseases, infections, and hospitalizations. Thus, kidney diseases are one of the most common diseases worldwide (3).

Antioxidants such as superoxide dismutase, catalase, glutathione reductase and glutathione-Stransferase are very important molecules which decrease free radicals (24).Reactive oxygen species (ROS) are superoxide radicals $\left(\mathrm{O}_{2}^{-}\right)$, hydrogen peroxide $\left(\mathrm{H}_{2} \mathrm{O}_{2}\right)$, hydroxyl radicals $\left(\mathrm{OH}^{-}\right)$and singlet oxygen which occur in small amounts during normal oxygen metabolism. Reactive oxygen species can initiate free radical chain reactions where various free radicals form (28). Activation of macrophage and neutrophils and the electron transport chain due to ultraviolet rays, metal catalyzed reactions, atmospheric pollution and inflammation are the factors that cause ROS production (29). 
Increase in oxygen molecules that can happen at certain levels is deactivated by the natural antioxidant molecules which are always present in certain amounts in the body. This allows the oxidant levels and the deactivation power of antioxidants in an organism to remain in balance (28). The delicate balance between free radicals and antioxidant defense system shifting in favor of prooxidant and oxidants, leads to the development of oxidative stress (30).

RONS are generated by all aerobic cells and play an important role in both aging and age-related diseases (4) They include radicals such as superoxide anion $\left(\mathrm{O}_{2}{ }^{\bullet-}\right)$, hydroxyl $(\mathrm{OH} \bullet)$, peroxyl $\left(\mathrm{RO}_{2} \bullet\right)$, nitric oxide $(\mathrm{NO} \bullet)$, singlet oxygen $\left({ }^{1} \mathrm{O}_{2}\right)$, nitrogen dioxide $\left(\mathrm{NO}_{2} \bullet\right.$ ) and lipidperoxyl (LOO•). These radicals are becoming increasingly implicated in human diseases. Non-radicals include hydrogen peroxide $\left(\mathrm{H}_{2} \mathrm{O}_{2}\right)$, hypochlorous acid $(\mathrm{HOCl})$, ozone $\left(\mathrm{O}_{3}\right)$, and peroxy nitrate $\left(\mathrm{ONOO}^{-}\right)$, nitrous acid $\left(\mathrm{HNO}_{2}\right)$, dinitrogen trioxide $\left(\mathrm{N}_{2} \mathrm{O}_{3}\right)$, lipid peroxide (LOOH) (5) .ROS show that they contain several carcinogens characteristics. Further, functional ROS concentrations may be required for normal cell functioning, and ROS is considered to be involved in plant and animal growth. Environmental or behavioral stressors (pollution, sunlight exposure, cigarette smoking, excessive alcohol consumption, chemicals/drugs, radiation etc.) or a tiny defect in antioxidant generation can cause a free radical excess known as "oxidative stress" and the dynamic redox balance between oxidants and antioxidants changes to oxidative potential

The Oxidative stress measurement could be done in both cases healthy and the one who has clinical conditions.In the case of renal failure (6). Directly and indirectly, oxidative stress will influence the kidney, for example, vascular reactivity, glomerular filtration, renal hemodynamics, and tubular reabsorption and secretion in all pieces of the nephron. Oxidative stress signaling changes all of these mechanisms during injury or disease and encourages mechanisms of cellular apoptosis, tissue injury, progression necrosis, fibrosis prevention, altered gene expression and unusual function of the kidney (7). Oxidative stress due to kidney damage related AKI occurring in the oxidation of numerous macromolecules (e.g., protein, DNA and lipid). Also OS in the occurrence of CKD, a section of nonhemodynamic factors play a role, either by glomerular damage and ischemia of the kidney are directly or related indirectly with hypertension, inflammation, and endothelial dysfunction, characteristics that stimulate excessive oxidative stress in CKD are including inflammation, malnutrition, excessive activity of oxidase, increased phagocytic activity, and decreased mechanisms of oxidative defense (8).

The aim of this study is to determine the relationship between superoxide dismutase (SOD), reduced glutathione (GSH), and catalase (CAT), and malondialdehyde (MDA) level in acute kidney failure and chronic kidney diseases.

\section{MATERIALS AND METHODS}

In this study, 31 patients were composed of acute kidney failure, 30 patients were chronic kidney failure and 31 patients were healthy individuals. The age range of sick and healthy individuals was between 18-65. Biochemical parameters were determined by serum samples. Before the collection of blood samples for this research, local ethical committee approval was obtained from Medical FacultyVan Yüzüncü Y1l University.From selected healthy and sick individuals $3 \mathrm{ml}$ blood was taken from venous blood sample and after that bloods were centrifuged with 5000r/min for 10 minutes and then serums were separated from plasma. The separated serums were used to determine the superoxide dismutase (SOD), reduced glutathione (GSH), catalase (CAT), and malondialdehyde (MDA) levels.

\section{DETERMINATION OF SUPEROXIDE DISMUTASE (SOD) ACTIVITY}

SOD activity was determined by using the proposed method of (9).SOD accelerates the dismutation of hydrogen peroxide and molecular oxygen of superoxide radicals $\left(\mathrm{O}_{2}{ }^{--}\right)$formed during the oxidative energy production. This method is based on the reading of topic density resulted from using of xanthine and xanthine oxidase in which superoxide radicals that generated from the blue colored formazan dye of the nitro blue tetrazolium (NBT) in the optical density wavelength of $560 \mathrm{~nm}$. The SOD that exists in the sample serum inhibits the formazan reaction by excluding superoxide radicals from the environment. Under the experimental conditions, 1 unit of SOD is the $\% 50$ inhibition of NBT reduction rate. 
$\%$ Inhibition $=[($ Blank OD - Sample OD $) /$ Blank OD $] \times 100$

\section{DETERMinATION OF CATALASE (CAT) ACTIVITY}

Catalase activity was determined according to the Aebi method in this study, which is used as a hydrogen peroxide substrate. The activity is carried out in the following manner, First, add $1.4 \mathrm{ml}$ of $30 \mathrm{mM} \mathrm{H}_{2} \mathrm{O}_{2}$ to the two tubesblind and add $0.1 \mathrm{ml}$ of phosphate buffer. Add $1.4 \mathrm{ml}$ of $30 \mathrm{mM} \mathrm{H}_{2} \mathrm{O}_{2}$ to the sample tube. Then $0.1 \mathrm{ml}$ of enzyme was added to the vortex. Absorbance's were read at $240 \mathrm{~nm}$ twice at 30 second intervals, and activity was thus determined (10).

Activity account:

Activity $=(2.3 / \Delta \mathrm{X}) \times[(\log \mathrm{A} 1 / \log \mathrm{A} 2)]$

$\Delta \mathrm{X}: 30$ seconds

2.3: $1 \mathrm{mmol}$ optical density of $\mathrm{H}_{2} \mathrm{O}_{2}$ in $1 \mathrm{~cm}$ light path.

\section{DETERMINATION OF REDUCED GLUTATHIONE (GSH) LEVEL}

The reduced glutathione (GSH) was measured as the final product of the reaction was achieved, that was the formation of the yellow color, of obtained clear liquid of sulfhydryl groups and DTNB $\left(5,5^{\prime}-(\right.$ dithiobis 2-nitrobenzoic acid). Measurement of the reduced glutathione level in the serum blood was done in $412 \mathrm{~nm}$ wavelength in the spectrophotometer (11).

Activity $(\mathrm{mg} / \mathrm{dl})=[(\mathrm{OD} 2-\mathrm{OD} 1) / 13600 \times \mathrm{E} 1 \mathrm{1.25}] \times 1000$

OD1: First absorbance before addition of DTNB at $412 \mathrm{~nm}$.

OD2: Second absorbance after addition of DTNB at 412nm.

E1: 1 in the calculations

13600 is the molar extinction coefficient of the yellow color that formed during the interaction of GSH and DTNB.

\section{DETERMINATION OF MALONDIALDEHYDE (MDA) LEVEL}

The reaction of fatty acids with free radicals result in malondialdehyde, which is the final product of lipid peroxidation, is measured with thibarbituric acid that gives a colored form (12). $200 \mathrm{ml}$ from the blood is taken and put into 1 tube $.800 \mathrm{ml}$ phosphate buffer, $25 \mathrm{ml}$ BHT solution, and $500 \mathrm{ml}$ of $\% 30$ TCA were added. The tubes were stirred with vortex and kept on ice for 2 hours. Then centrifuged at $2000 \mathrm{rpm}$ for 15 minutes, $1 \mathrm{ml}$ from supernatant was taken and transferred to other tubes. Then $75 \mathrm{ml}$ of EDTA and 250ml of TBA were added. Tubes were mixed in the vortex and kept in a hot water bath for 15 minutes. Then, they were brought to room temperature and their absorbance was read at UV/V spectrophotometer at $532 \mathrm{~nm}$.

Calculation of malondialdehyde level:

$\mathrm{C}=\mathrm{F} \times 6.41 \times \mathrm{A}$

C: Concentration

F: Dilution factor

\section{A: Absorbance}

\section{STATISTICAL ANALYSIS}

Descriptive statistics for the study parameters were expressed as mean, standard deviation, minimum and maximum values. One-way analysis of variance (ANOVA) was used in cases with normal distribution condition. Kruskalwallis test was used in cases where normal distribution condition was not provided.The statistical significance level was taken as $\mathrm{p}<0.05$ and the SPSS statistical software version 19.0 (SPSS Inc, Chicago, III, USA) pack has used for analyses. 


\section{RESULTS}

Descriptive statistics and comparison results for SOD, CAT, GSH and MDA are given in Table 1. When Table 1 is examined, the difference between the group averages for SOD, CAT, GSH and MDA was found statistically significant $(\mathrm{p}<0.05)$. SOD, CAT and GSH were high in the control group, were low in patients acute and chronic (Figure 1,2,3). Whereas MDA was found low in the control group, it was found high in Acute and Chronic patients (Figure 1).

Table1. Comparison between the groups

\begin{tabular}{|l|c|c|c|c|}
\hline Parameters & $\begin{array}{c}\text { Controls } \\
(\mathrm{n}=31) \text { Mean } \pm \text { SD }\end{array}$ & $\begin{array}{c}\text { Patients acute } \\
(\mathrm{n}=31) \text { Mean } \pm \text { SD }\end{array}$ & $\begin{array}{c}\text { Patients chronic } \\
(\mathrm{n}=30) \text { Mean } \pm \text { SD }\end{array}$ & $\mathrm{p}$ \\
\hline $\mathrm{SOD}(\mathrm{U} / \mathrm{L})$ & $7.351 \pm 0.381$ & $1.312 \pm 0.754$ & $2.012 \pm 0.661$ & 0.001 \\
\hline $\mathrm{CAT}(\mathrm{U} / \mathrm{L})$ & $0.415 \pm 0.124$ & $0.075 \pm 0.005$ & $0.077 \pm 0.006$ & 0.001 \\
\hline $\mathrm{GSH}(\mu \mathrm{mol} / \mathrm{L})$ & $2.8 \times 10-4 \pm 6.5 \times 10^{-5}$ & $2.1 \times 10-5 \pm 1.6 \times 10^{-5}$ & $6.1 \times 10-6 \pm 5.3 \times 10^{-6}$ & 0.001 \\
\hline $\mathrm{MDA}(\mu \mathrm{mol} / \mathrm{L})$ & $0.708 \pm 0.065$ & $2.155 \pm 0.753$ & $1.921 \pm 0.497$ & 0.001 \\
\hline
\end{tabular}

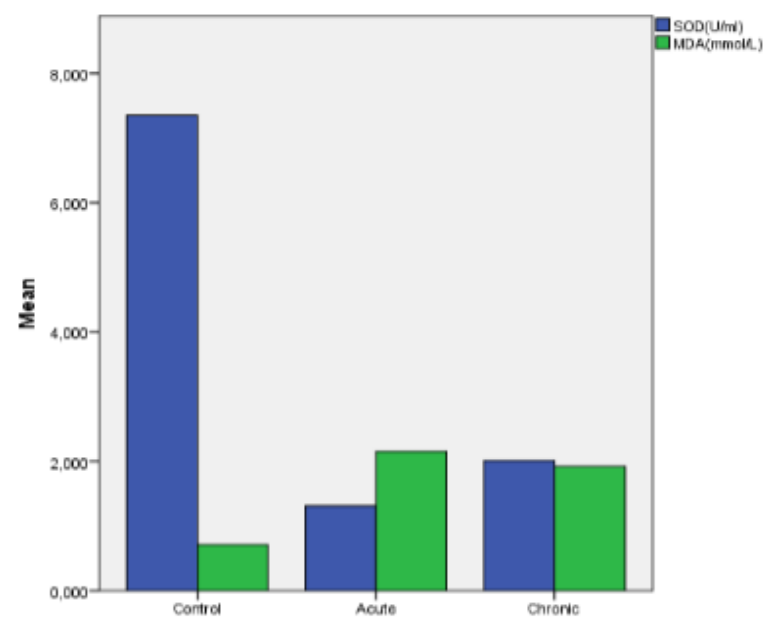

Figure1. SOD and MDA levels among between groups

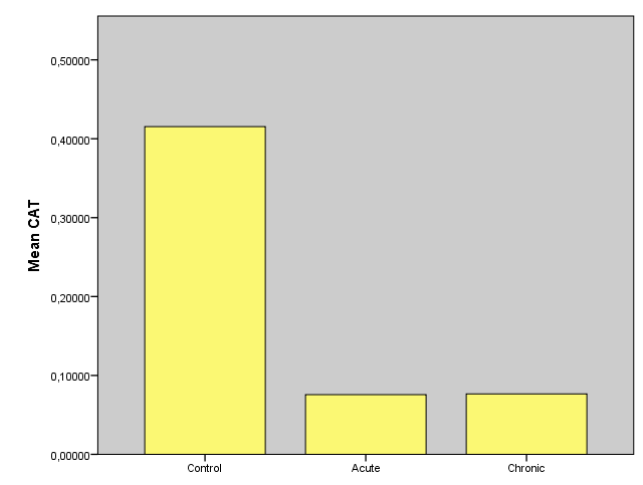

Figure2. CAT activity among between groups

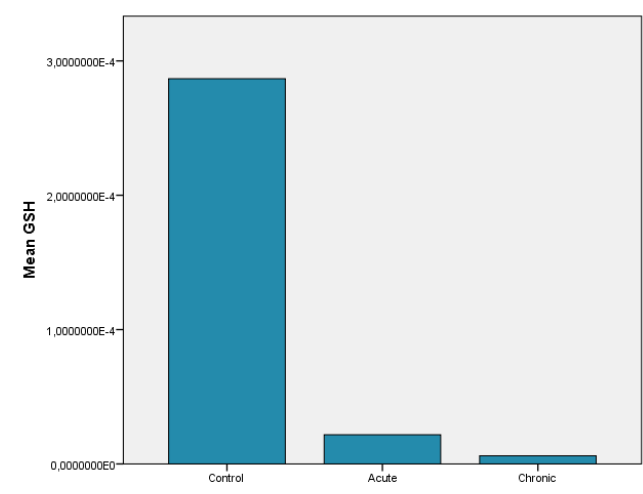

Figure3.GSH levels among between groups 


\section{DISCUSSION}

Normally the tissue damage caused by oxidants in the body is controlled by enzymatic and nonenzymatic antioxidant defense systems. The most important antioxidant enzymes are SOD, GHPx, and CAT; among the nonenzymatic antioxidants are glutathione, tocopherole (vitamin E), ascorbic acid (vitamin C), carotene (vitamin A), albumin, bilirubin and uric acid (26). Oxidative stress is associated with decreases in antioxidants or with increases in the production of oxidants; this situation, by the peroxidation of phospholipids, causes damage in the vital substances of the body such as lipids, proteins and DNA (27).

In the case of renal failure, ROS will attack cell membranes, thus causes the change in the antioxidant enzymatic mechanism, and lipid peroxidation products such as MDA (6). It is suggested that ROS play an important role in the initiation and progression of kidney diseases and injuries, such as diabetic nephropathy, and hypertension. While they will produce in several tissues, also cell pressures in both renal and vascular cells. In the kidney and vascular system, there are many sources of ROS and RNS, which may present in ordinary and disease conditions, while mitochondrial and NADPH oxidase cumulating is the major factor of oxidative stress in the kidney (13).

Impairment of antioxidant defense leads to oxidative stress that can lead to complications such as those observed in chronic kidney disease (CKD) and acute kidney injury (AKI). Insights into kidney damage caused by oxidative stress can be acquired by observing the effects of sepsis proinflammatory processes on this organ. This disease causes serious tubule interstitial damage to fast and progressive renal disease. The existence of pro-inflammatory ROS, cytokines and apoptosis due to interstitial tubular dysfunction and loss of renal epithelial cells by damage renal tubular injury, That, in turn, is closely tied to the microvascular structure and renal blood flow that affect functional modification.Oxidative stress due to kidney damage related AKI occurring in the oxidation of numerous macromolecules (e.g., protein, DNA and lipid). Malondialdehyde (MDA) and 4hydroxynonenics are in large products in Lipid peroxidation production (8). Also, OS in the occurrence of CKD, a section of non-hemodynamic factors play a role, either by glomerular damage and ischemia of the kidney are directly or related indirectly with hypertension, inflammation, and endothelial dysfunction, characteristics that stimulate excessive oxidative stress in CKD are including inflammation, malnutrition, excessive activity of oxidase, increased phagocytic activity, and decreased mechanisms of oxidative defense.

One of the most sensitive organs that influenced by ROS regarded to be a kidney, because it is rich in blood supply, vascular tissues, and the organ that has high metabolic activity. OS could be dramatically increased in the CKD cases of hemodialysis before and after compared with the control group. It is reported that in the CKD cases, OS magnitudes are extremely high. For instance, patients who have CKD cases there OS level were three times higher. Furthermore, many researchers noted extremely high levels of MDA in pre- and post-dialysis cases compared to controls (14).MDA levels in patients with acute and chronic renal failure were found to be significantly increased compared to the control group $(\mathrm{p}<0.05)$. It is reported that there are many factors which influencing oxidative stress in renal failure, such as increased production of oxidative metabolism agents, and reduced defense of antioxidants (15).

The activity of SOD was examined, the result showed that its activity significantly was decreased $(p<0.05)$ among acute and chronic renal patients when compared to the control group. When serum MDA and SOD levels were compared in patients with acute and chronic it was also noted that there was a significant increase in serum MDA of renal failure and decreased SOD in renal failure patients. SOD is the first defense line from front against superoxide anions and turns it into hydrogen peroxide. Several studies indicate that the concentration of MDA, a lipid peroxidation by-product, is significantly increased in patients with CKD before initiation of dialysis and renal replacement therapy compared to the control group (16). In this research, there was a rise in MDA concentrations in patients with CKD. With the severity of kidney dysfunction, this indicates that these patients have raised lipid peroxidation.

OS causes endothelial dysfunction and decreasing in plasma SOD. Therefore, many studies have shown that SOD and other antioxidant enzymes are reduced in pre-clinical AKI cases, and genetic 
impairment of SOD increases sensitivity to AKI in ischemia and hypoxia (17). One of the factors which lead to elevate of super oxide dismutase activity is over proliferation of $\mathrm{O}_{2}^{-}$macrophages reducing antioxidant plasma activity in patients with CKD may be contributing to higher oxidative damage and generation of renal problems (15).

The OS could be lead to higher levels with CKD patients, which that vital characteristics of renal patients such as diabetes, hypertension, and advanced age, all of them increased OS compared to healthy. Also, another source of OS with CKD cases is inflammation. It is known that, there is a wide connection between kidney disease and indicators as well as mediators such as interleukin (IL)-6, Creactive protein, fibrinogen, and tumor necrosis factor- $\alpha$. Those were shown as an increased level of MDA concentrations and were detected serum SOD level decreased (18).

Additional investigations have determined as the level of MDA is significantly more elevated and antioxidant parameters levels have reduced than normal control range, such as CAT activity. In gastric, kidney, breast, lung cancer and colorectal adenomas, oxidative stress alike to increase in MDA and antioxidant parameter levels decreased.There are situations in which plasma MDA will increase such as elevates alcohol, saturated fat and consumption of meat present. However it is known that high fiber nutrition is reversely proportionate to MDA (19).The increase in serum MDA indicated that OS could happen in free radical convinced in the cell membrane of lipid component, therefore MDA is work as a good indicator for detecting oxidative stress in degenerative disease such as CKD. The level of SOD enhanced is trying to fight oxidative stress. The early marker of acute renal failure is SOD activity (7).

Meanwhile this research, the resulting activity of catalase (CAT) was statistically and significantly decreased when it is compared to the control group in acute and chronic renal failure patients $(\mathrm{P}<0.05)$. It is known that inside of the kidney there are aerobic cells in which they contain high amount of catalase. CAT has an important role in reducing ROS as well as it leads to inhibition of lipid peroxidation. The failure of mitochondrial function comes from catalase defection, which leads to accumulation of ROS inside of mitochondria and results of mitochondrial disfunction (7). The two important groups of antioxidant enzymes which they are glutathione peroxidase and catalase contribute in oxidation of $\mathrm{H}_{2} \mathrm{O}_{2}$ and results in water molecule and oxygen. Glutathione peroxidase comes in first line importance, while catalase comes secondly in prevention against $\mathrm{H}_{2} \mathrm{O}_{2}$ formation process. Both enzymes works together in significant way, in order to metabolizing of $\mathrm{H}_{2} \mathrm{O}_{2}$ that may formed during normal metabolism process, and in kidney disease circumstances (20). Catalase plays a major role in decreasing ROS and avoiding lipid peroxidation. Catalase deficiency results in accumulation of mitochondrial ROS, an impact that causes to structural impairment of mitochondria. Low levels of hydrogen peroxide lead to the maintenance of basal tone in vascular bodies throughout the body, including the kidneys, its removal impairing ordinary hemodynamic function (7).SOD and CAT activity are reduced by oxidative damage. That defect promotes kidney disease after acute renal ischemia. AKI is protective against oxidation and/or renal damage via endogenous or nutritional antioxidants. For instance, vitamin $\mathrm{E}$ and selenium (which can increase the activity of antioxidant enzymes dependent on GSH) reduce nephrotoxicity (17).

In this study, the activity of GSH was statistically and significantly decreased when it is compared to the control groups in acute and chronic renal failure $(\mathrm{P}<0.05)$. GSH plays a key role in cellular resistance against oxidative damage. In this study, GSH levels were significantly decreased in the patients of acute and chronic when compared with a group of healthy individuals. The results indicate that chronic renal and acute renal patients have lower GSH levels.It appears that renal GSH content can be altered by modifying the stress of renal oxidant. When renal work (and therefore the consumption of renal oxygen) was reduced by either reducing the filtered sodium load as in the renal artery or by pharmacologically inhibiting renal sodium reabsorption in the entire nephron, facing a constantly filtered sodium load, renal GSH concentrations rose. We assume that renal GSH rose because the reduction in renal $\mathrm{O}_{2}$ consumption that would follow the reduction in renal sodium reabsorption resulted in less oxygen-free radicals being formed. Less GSH was absorbed to protect the cell against these free radicals and therefore GSH levels rose (21).

The kidney damage one of the factored by reducing lipid peroxidation and the antioxidant enzymes could increase the level of the protection system's scavenging ability. Antioxidants have been work 
such as increasing the level of activity of SOD to protect the kidney and decreasing ROS generation as well as reducing MDA. For example one of the antioxidants that were found in medicinal plants such as Troxerutin has richly amount in cereal grain, tea, coffee, and fruit, and vegetable varieties work to increase antioxidant activity such as the level of SOD and CAT to decrease the level of MDA (22). Antioxidants function by preventing free radical formation. According to the literature, studies have shown that antioxidants reduce the effects of free radicals and prevent cell damage (25). The cell protected membrane by vitamin $\mathrm{E}$ against lipid peroxidation when making a low radical sensitivity tocopherol, with $\mathrm{O}_{2}$ and hydroxyl radical such as vitamin $\mathrm{C}$ scavenges. Vit $\mathrm{E}$ composed in the main compound as well as alpha and gamma-tocopherol there has increased level of antioxidant activity. Some antioxidants such Vit E, Vit $\mathrm{C}$ and $\beta$-carotene uses as decreased the level of serum 8-oxo2'deoxyguanosine when uses for 1 month like the indicator of DNA damage in kidney failure (23).

\section{Conclusions}

This study showed that oxidative stress levels increased in kidney patients, whereas antioxidant activities such as SOD, CAT and GSH decreased. These results show that kidney failure is associated with oxidative stress. The results of our study are compatible with the results of the literature.Increasing antioxidant levels and decreasing the MDA enzyme level can help protect the kidney from oxidative stress. This study is the first one to show the relationships of MDA, SOD, CAT and GSH in acute kidney failure and chronic kidney diseases.As a result, we think that more studies are needed on this subject.

\section{REFERENCES}

[1] Stevens, L. A., \& Levey, A. S. (2009). Measured GFR as a confirmatory test for estimated GFR. Journal of the American Society of Nephrology, 20(11), 2305-2313.

[2] Bellomo, R., Kellum, J. A., Ronco, C., 2012. Acute kidney injury. The Lancet, 380(9843) : 756-766.

[3] Richardson, J. (2017). The Gypsy debate: Can discourse control?. Andrews UK Limited.

[4] Powers, B. G., Carlisle, J. A., \& Kuba, L. M. (2011). U.S. Patent Application No. 12/906,077.

[5] Kumar, J. A., \& Ganesh, L. S. (2009). Research on knowledge transfer in organizations: a morphology. Journal of knowledge management.

[6] Montazerifar, F., Hashemi, M., Karajibani, M., Sanadgol, H., Dikshit, M., 2012. Evaluation of lipid peroxidation and erythrocyte glutathione peroxidase and superoxide dismutase in hemodialysis patients. Saudi Journal of Kidney Diseases and Transplantation, 23(2): 274-279.

[7] Ratliff, B. B., Abdulmahdi, W., Pawar, R., Wolin, M. S., 2016. Oxidant mechanisms in renal injury and disease. Antioxidants and Redox Signaling, 25(3): 119-146.

[8] Cristol, S. M., Alfonso, E. C., Guildford, J. H., Roussel, T. J., \& Culbertson, W. W. (1996). Results of large penetrating keratoplasty in microbial keratitis. Cornea, 15(6), 571-576.

[9] Popov, B., Gadjeva, V., Valkanov, P., Popova, S., Tolekova, A., 2003. Lipid peroxidation, superoxide dismutase and catalase activities in brain tumor tissues. Archives of Physiology and Biochemistry, 111(5): 455-459.

[10] Aebi, H., 1984. Catalase in vitro. In Methods in enzymology, 105:121-126.

[11] Beutler, E., 1963. Improved method for the determination of blood glutathione. J. lab. clin. Med, 61: 882888.Biochemistry, 70(11): 1251-1255.

[12] Gutteridge, J. M., 1995. Lipid peroxidation and antioxidants as biomarkers of tissue damage. Clinical Chemistry, 41(12): 1819-1828.

[13] Brodsky, S. V., Gao, S., Li, H., Goligorsky, M. S., Hyperglycemic switch from mitochondrial nitric oxide to superoxide production in endothelial cells. Am J Physiol Heart Circ Physiol, 283: 2130-2139.

[14] Nagamma, T., Ahmed, S., Pai, A., Mohan, S., Chathuvedi, A., Singh, P. P., 2014. Evaluation of oxidative stress and antioxidant activity in pre and post hemodialysis in chronic renal failure patients from Western region of Nepal. Bangladesh Journal of Medical Science, 13(1): 40-44.

[15] Hacsşevki, A., 2008. Effect of hemodialysis on oxidative stress in patients with chronic renal failure. Ankara Üniversitesi Eczaculk Fakültesi Dergisi, 37(2): 91-100.

[16] Eleftheriadis, T., Kartsios, C., Yiannaki, E., Kazila, P., Antoniadi, G., Liakopoulos, V., \& Markala, D. (2008). Chronic inflammation and T cell zeta-chain downregulation in hemodialysis patients. American journal of nephrology, 28(1), 152-157. 
Determination of Oxidative Stress Levels and Some Antioxidant Activities in Acute and Chronic Renal Failure Patients

[17] Dennis, J., Witting, P., 2017. Protective role for antioxidants in acute kidney disease. Nutrients, 9(7): 718725

[18] Krata, N., Zagożdżon, R., Foroncewicz, B., Mucha, K., 2018. Oxidative stress in kidney diseases: the cause or the consequence?. Archivum Immunologiae Et Therapiae Experimentalis, 66(3): 211-220.

[19] Didžiapetrienė, J., Bublevič, J., Smailytė, G., Kazbarienė, B., Stukas, R., 2014. Significance of blood serum catalase activity and malondialdehyde level for survival prognosis of ovarian cancer patients. Medicina, 50(4): 204-208.

[20] Kobayashi, M., Sugiyama, H., Wang, D. H., Toda, N., Maeshima, Y., Yamasaki, Y., Makino, H., 2005. Catalase deficiency renders remnant kidneys more susceptible to oxidant tissue injury and renal fibrosis in mice. Kidney International, 68(3): 1018-1031.

[21] Weinberg, J. M., \& Davis, J. A. 1987. Protective effects of glycine against hypoxic tubule cell injury are independent of the presence of butyrate. In CLINICAL RESEARCH 35(3): 559-559.

[22] Rafieian-kopaei, M., 2013. Medicinal plants for renal injury prevention. Journal of Renal Injury Prevention, 2(2): 63-65.

[23] Modaresi, A., Nafar, M., Sahraei, Z., 2015. Oxidative stress in chronic kidney disease. Iranian Journal of Kidney Diseases, 9(3): 165-179.

[24] Turan, K., Uckan, K., Sarikaya, E., Demir, H., \& Demir, C. (2017). Investigation of prolidase, adenosine deaminase, glutathione s-transferase and glutathione reductase activities in patients with abortus imminens. JOURNAL OF CLINICAL AND ANALYTICAL MEDICINE, 8(6), 519-522.

[25] Poston, L., \& Raijmakers, M. T. M. (2004). Trophoblast oxidative stress, antioxidants and pregnancy outcome-a review. Placenta, 25, S72-S78.

[26] Valko, M., Leibfritz, D., Moncol, J., Cronin, M. T., Mazur, M., \& Telser, J. (2007). Free radicals and antioxidants in normal physiological functions and human disease. The international journal of biochemistry \& cell biology, 39(1), 44-84.

[27] Garça, M. F., Turan, M., Avşar, B., Kalkan, F., Demir, H., Kozan, A., \& Bozan, N. (2015). The evaluation of oxidative stress in the serum and tissue specimens of patients with chronic otitis media. Clinical and experimental otorhinolaryngology, 8(2), 97.

[28] B. Halliwell, "Reactive Oxygen Species in Living Systems: Source, Biochemistry and Role in Human Disease," American Journal of Medicine, Vol. 91, No. 3C, 1991, pp.14-22

[29] E. Cadenas, "Biochemistry of Oxygen Toxicity," Annual Review of Biochemistry, Vol. 58, 1989, pp. 79110

[30] Arslan, A., Demir, H., Ozbay, M. F., \& Arslan, H. (2014). Evaluation of lipid peroxidation and some antioxidant activities in patients with primary and metastatic liver cancer. Journal of Cancer Therapy, 2014.

Citation: Seerwan Hamadameen SULAIMAN et al." Determination of Oxidative Stress Levels and Some Antioxidant Activities in Acute and Chronic Renal Failure Patients", International Journal of Clinical Chemistry and Laboratory Medicine (IJCCLM), vol. 7, no.1, pp. 12-19, 2021. http://dx.doi.org/10.20431/ 2455-7153.0701002

Copyright: (C) 2021 Authors. This is an open-access article distributed under the terms of the Creative Commons Attribution License, which permits unrestricted use, distribution, and reproduction in any medium, provided the original author and source are credited. 